\title{
On Lower Semicontinuity and Metric Upper Semicontinuity of Nemytskii Set-Valued Operators
}

\author{
S. Rolewicz and Song Wen
}

\begin{abstract}
Sufficient conditions of lower semicontinuity and metric upper semicontinuity of Nemytskii set-valued operators $N_{F}$ generated by a set-valued function $F: \Omega \times X \rightarrow 2^{Y}$, where $X$ and $Y$ are Orlicz-Musielak $F$-spaces are presented.
\end{abstract}

Keywords: Nemytskii set-valued operators, lower semicontinuity, metric upper semicontinuity, superposition measurable set-valued operators

AMS subject classification: $47 \mathrm{H} 04,28 \mathrm{C} 20,54 \mathrm{C} 60$

In years 1933 - $1934 \mathrm{~V}$. Nemytskii (see [10] and [11]) considered the operator $F$ : $L^{2}[a, b] \rightarrow L^{2}[a, b]$ defined by $y(\cdot)=N_{F}(x(\cdot))$, where $y(t)=F(t, x(t))$. From that time the operator $N_{F}$ was generalized in several way and there is a lot of papers devoted to such subjects. Operators of this type are now called Nemytskiz operators.

In last years new important applications of Nemytskii set-valued operators in the theory of differential and integral inclusions appear (see [1 - 4]). For these applications lower semicontinuity and metric upper semicontinuity of Nemytskii set-valued operators are important.

In [1] Appell, Ngyen and Zabrejko give sufficient conditions of lower semicontinuity of Nemytskii set-valued operators acting in so-called ideal spaces. We shall not give the definition here. We want only to mention that ideal spaces are some spaces of functions defined on a measure space $\Omega$ admitting values in finite-dimensional spaces and it can be shown that each Orlicz space admitting values in a finite-dimensional space is an ideal space.

Thus the natural problem arose to give sufficient conditions of lower semicontinuity and metric upper semicontinuity of Nemytskii set-valued operators for spaces consisting of functions admitting values in infinite dimensional spaces.

In this paper sufficient conditions of lower semicontinuity and metric upper semicontinuity of Nemytskii set-valued operators acting in Orlicz-Musielak $F$-spaces are

S. Rolewicz: Polish Acad. Sci., Inst. Math., ul. Sniadeckich 8, skr. poszt. 137, P - 00-950 Warszawa. The paper is partially supported by the Polish Committee for Scientific Research under Grant No. 220099102.

Song Wen: Harbin Normal University, Department of Mathematics, Harbin, China 
given.

Let $X$ be a separable $F$-space (i.e. a complete linear metric space; basic properties can be found in [12]) with an $F$-norm $\|\cdot\|_{X}$ and $(\Omega, \Sigma, \mu)$ a measure space with complete and $\sigma$-finite measue $\mu$. A function $x=x(t)$ mapping $\Omega$ into $X$ is called measurable if for every open set $Q \subset X$ the inverse image $x^{-1}(Q)=\{t \in \Omega: x(t) \in Q\}$ is measurable, i.e. if $x^{-1}(Q) \in \Sigma$. The set of all measurable functions defined on $\Omega$ with values in $X$ we shall denote by $S(\Omega, X)$.

A closed-valued set-valued function $F=F(t)$ mapping $\Omega$ into subsets of $X$ is called measurable if for every open set $Q \subset X$ the inverse image $F^{-1}(Q)=\{t \in \Omega$ : $F(t) \cap Q \neq \emptyset\}$ is measurable, i.e. if $F^{-1}(Q) \in \Sigma$. By a measurable selection of the set-valued function $F$ we mean a (single-valued) function $x_{F}$ such that $x_{F}(t) \in F(t)$ for all $t \in \Omega$.

Let $F=F(t, x)$ be a closed-valued set-valued function mapping $\Omega \times X$ into subsets of an $F$-space $Y$, i.e. into $2^{Y}$. We say that $F$ is sup-measurable if for any measurable function $x(\cdot): \Omega \rightarrow X$ the set-valued function $s \rightarrow F(s, x(s)): \Omega \rightarrow 2^{Y}$ is measurable.

Given a sup-measurable closed-valued set-valued function $F: \Omega \times X \rightarrow 2^{Y}$. This set-valued function induces a set-valued operator $N_{F}: S(\Omega, X) \rightarrow S(\Omega, Y)$ defined by $y(\cdot)=N_{F}(x(\cdot))$, where $y(t)=F(t, x(t))$. The set-valued operator $N_{F}$ is called superposition operator (or Nemytskis operator) generated by the set-valued function $F$.

Let $N=N(t, u)$ be a real-valued measurable function defined on $\Omega \times R$ such that for every $t \in \Omega$ the function $N(t, \cdot)$ is increasing and moreover $N(t, 0)=0$ for all $t \in \Omega$. Then we can define on $S(\Omega, X)$ a metrizing modular

$$
\rho_{N, \mu}(x(\cdot))=\int_{\Omega} N\left(t,\|x(t)\|_{X}\right) d \mu
$$

(see, e.c., Nakano ([7], [8: p. 153] and [9: p. 204]), Musielak [5: p. 1] and Rolewicz [12: p. 6]). The set of those measurable functions $x(\cdot) \in S(\Omega, X)$ that there is a positive $k$ such that $\rho_{N, \mu}(k x(\cdot))<\infty$ we shall denote by $N(L(\Omega, \Sigma, \mu ; X))$. Recall that a metrizing modular on a linear space $X$ is a function $\rho: X \rightarrow[0, \infty]$ having the following properties:

(md1) $\rho(x)=0$ if and only if $x=0$

(md2) $\rho(a x)=\rho(x)$ provided $|a|=1$

(md3) $\rho(a x+b y) \leq \rho(x)+\rho(y)$ provided $a, b>0$ and $a+b=1$

(md4) $\rho\left(a_{n} x\right) \rightarrow 0$ provided $a_{n} \rightarrow 0$ and $\rho(x)<+\infty$

(md5) $\rho\left(a x_{n}\right) \rightarrow$ provided $\rho\left(x_{n}\right) \rightarrow 0$.

We shall denote by $(X, \rho)$ a linear space with a modular $\rho$ and we shall call it modular space. Let $(X, \rho)$ be a modular space with metrizing modular $\rho$. It is known that $\rho$ induces in the space $X$ an $F$-norm $\|\cdot\| x$ by

$$
\|x\|_{X}=\inf \left\{\varepsilon>0 \mid \rho\left(\frac{x}{\varepsilon}\right)<\varepsilon\right\} .
$$


The norm $\|\cdot\| x$ is equivalent to the moduler $\rho$ in the sense that, for any sequence $\left\{x_{n}\right\},\left\|x_{n}\right\| x \rightarrow 0$ if and only if $\rho\left(x_{n}\right) \rightarrow 0$ (see Musielak and Orlicz [6] and Musielak [5: p. 2]; see also Rolewicz [12: p. 8]). Observe that if the functions $x_{n}(\cdot) \in$ $N(L(\Omega, \Sigma, \mu ; X)) \quad(n \in \mathbb{N})$ have disjoint supports, then

$$
\sum_{n=1}^{\infty} \rho_{N, \mu}\left(x_{n}(\cdot)\right)=\rho_{N, \mu}\left(\sum_{n=1}^{\infty} x_{n}(\cdot)\right) .
$$

Let $\left(X, \rho_{X}\right)$ and $\left(Y, \rho_{Y}\right)$ be two modular spaces. A set-valued operator $\Gamma=\Gamma(x)$ mapping $\left(X, \rho_{X}\right)$ into subsets of $\left(Y, \rho_{Y}\right)$ will be called lower semicontinuous at a point $\left(x_{0}, y_{0}\right) \in X \times Y$ if it is lower semicontinuous at this point in the metric induced by the $F$ norms introduced above. In other words $\Gamma$ is lower semicontinuous at $\left(x_{0}, y_{0}\right)$ if for every $r>0$ there is a number $q_{\left(z_{0}, y_{0}\right)}(r)>0$ with the following property: for every $x \in X$ such that $\rho_{N, \mu}\left(x_{0}(\cdot)-x(\cdot)\right)<q_{\left(x_{0}, y_{0}\right)}(r)$ there is an $y \in \Gamma(x)$ such that $\rho_{M, \mu}\left(y_{0}(\cdot)-y(\cdot)\right)<r$. This is equivalent with the property that $\rho_{N, \mu}\left(x_{0}(\cdot)-x(\cdot)\right)<q_{\left(x_{0}, y_{0}\right)}(r)$ implies

$$
\Gamma(x) \cap Q \neq \emptyset
$$

where $Q=\left\{y: \rho_{M, \mu}\left(y_{0}(\cdot)-y(\cdot)\right)<r\right\}$.

A set-valued operator $\Gamma$ mapping $\left(X, \rho_{X}\right)$ into subsets of $\left(Y, \rho_{Y}\right)$ will be called lower semicontinuous at a point $x_{0} \in X$ if it is lower semicontinuous at all the points $\left(x_{0}, y_{0}\right) \quad\left(y_{0} \in \Gamma\left(x_{0}\right)\right)$.

Now we shall introduce a notion of global lower semicontinuity at $x_{0}$, which is nothing else than an uniformization of lower semicontinuity on $\Gamma\left(x_{0}\right)$. More precisely, we say that a set-valued mapping $\Gamma$ is globally lower semicontinuous at the point $x_{0}$, if for every $r>0$ there is a $q_{x_{0}}(r)>0$ with the following property: for every $y_{0} \in \Gamma\left(x_{0}\right)$ and for every $x \in X$ such that $\rho_{N_{, \mu}}\left(x_{0}(\cdot)-x(\cdot)\right)<q_{x_{0}}(r)$ there is an $y \in \Gamma(x)$ such that $\rho_{M, \mu}\left(y_{0}-y\right)<r$. This is equivalent with the property that $\rho_{N, \mu}\left(x_{0}(\cdot)-x(\cdot)\right)<q_{x_{0}}(r)$ implies

$$
\Gamma\left(x_{0}\right) \subset B(\Gamma(x), r)
$$

where

$$
B(A, s)=\left\{y(\cdot): \inf _{y_{1}(\cdot) \in A} \rho_{M, \mu}\left(y_{1}(\cdot)-y(\cdot)\right)<s\right\} .
$$

The essential difference between lower semicontinuity and global lower semicontinuity is that in the first case $q_{\left(x_{0}, y_{0}\right)}>0$ depends on $x_{0}$ and $y_{0} \in \Gamma\left(x_{0}\right)$, while in the second case it depends on $x_{0}$ only.

Changing the role of $x$ and $x_{0}$ in formula (5) we obtain a notion of metric upper semicontinuity. We say that the set-valued mapping $\Gamma$ is metric upper semicontinuous (Hausdorff upper semicontinuous) at a point $x_{0}$ if for every $r>0$ there is a $q_{x_{0}}(r)>0$ such that $\rho_{N, \mu}\left(x_{0}(\cdot)-x(\cdot)\right)<q_{x_{0}}(r)$ implies

$$
\Gamma(x) \subset B\left(\Gamma\left(x_{0}\right), r\right) .
$$

The both notions are not equivalent. Indeed, it is easy to give an example of a set-valued mapping $\Gamma$ which is globally lower semicontinuous at a point $x_{0}$, but which is 
not metric upper semicontinuous at this point. Conversely, an example of a set-valued mapping $\Gamma$ which is metric upper semicontinuous at a point $x_{0}$ but which is not globally lower semicontinuous at this point can be easily given too.

In the sequel we shall add some assumptions about the functions $N(t, u)$ and $M(t, u)$. Namely, we assume that the function $N=N(t, u)$ satisfies the following condition

(A) For every $\varepsilon>0$ there are numbers $\alpha>0$ and $\delta>0$ such that for every measurable set $E \subset \Omega$ with $\mu(E)>\delta$ we have $\int_{E} N(t, \varepsilon) d t \geq \alpha$.

Observe that in Orlicz spaces, i.e. in the case when $N(t, u)=N_{0}(u)$ depends only on $u$, the condition (A) is satisfied. Further, we assume that the function $M(t, u)$ satisfies the following condition

$\left(\Delta_{2}\right)$ There are a constant $k \geq 1$ and a non-negative function $\delta$ such that $\int_{E} M(t, \delta(t)) d \mu$ $<+\infty$ and for almost all $t$, if $u \geq \delta(t)$, then $M(t, 2 u) \leq k M(t, u)$.

The condition $\left(\Delta_{2}\right)$ plays an essential role in the theory of Orlicz-Musielak spaces. Observe that if the function $M(t, u)$ satisfies condition $\left(\Delta_{2}\right)$, then from the fact that $\rho_{M, \mu}(x)<+\infty$ and $\rho_{M, \mu}(y)<+\infty$ it follows that $\rho_{M, \mu}(a x+b y)<+\infty$ for all real $a$ and $b$. We conclude that $M(L(\Omega, \Sigma, \mu ; Y))$ is the set of those measurable functions $y(\cdot)$ with values in the space $X$, that $\rho_{M, \mu}(y)<+\infty$ (see [5: p. 52] and [6]).

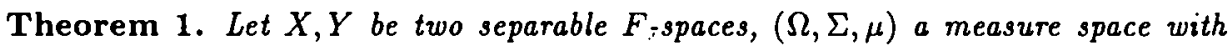
complete non-atomic and $\sigma$-finite measure $\mu$, and $N_{F}$ a Nemytskiz set-valued operator mapping of a modular space $\left(N(L(\Omega, \Sigma, \mu ; X)), \rho_{N, \mu}\right)$ into subsets of a modular space $\left(M(L(\Omega, \Sigma, \mu ; Y)), \rho_{M, \mu}\right)$ induced by a sup-measurable set-valued function $F(t, u): \Omega \times$ $X \rightarrow 2^{Y}$. Suppose that

(i) the function $N=N(t, u)$ satisfies condition (A)

(ii) the function $M=M(t, u)$ satisfies condition $\left(\Delta_{2}\right)$.

If the function $=F(t, u)$ is globally lower semicontinuous with respect to $u$ for almost all $t \in \Omega$, then the operator $N_{F}$ is globally lower semicontinuous.

Proof. Suppose that the operator $N_{F}$ is not globally lower semicontinuous. This implies that there are a number $r>0$ and sequences $\left\{x_{n}(\cdot)\right\},\left\{y_{n}(\cdot)\right\}$ with $y_{n}(\cdot) \in$ $N_{F}\left(x_{0}(\cdot)\right)$ such that

$$
\begin{aligned}
& \rho_{N, \mu}\left(x_{n}(\cdot)-x_{0}(\cdot)\right) \rightarrow 0 \\
& \inf _{z(\cdot) \in N_{F}\left(x_{n}(\cdot)\right)} \rho_{M, \mu}\left(z(\cdot)-y_{n}(\cdot)\right)>r \quad(n \in N) .
\end{aligned}
$$

By [2: Theorem 8.24 and Corollary 8.23], for arbitrary $\eta>0$ there is a sequence $\left\{z_{n}(\cdot)\right\}$ of measurable selections $z_{n}(\cdot) \in N_{F}\left(x_{n}(\cdot)\right)$ such that, for almost all $t \in \Omega$,

$$
\left\|y_{n}(t)-z_{n}(t)\right\|_{Y}<(1+\eta) d_{Y}\left(y_{n}(t), F\left(t, x_{n}(t)\right)\right)
$$


where $d_{Y}(y, A)=\inf _{z \in A}\|z-y\|_{Y}$ denotes the distance of a point $y$ to a set $A$ in the norm $\|\cdot\|_{Y}$. We denote $u_{n}(t)=\left\|y_{n}(t)-z_{n}(t)\right\|_{Y}$. Since $z_{n}(\cdot) \in N_{F}\left(x_{n}(\cdot)\right)$, then

$$
\int_{\Omega} M\left(t, u_{n}(t)\right) d \mu \geq \inf _{z(\cdot) \in N_{F}\left(x_{n}(\cdot)\right)} \rho_{M, \mu}\left(z(\cdot)-y_{n}(\cdot)\right)>r \quad(n \in \mathbb{N}) .
$$

The convergence $\rho_{N, \mu}\left(x_{n}(\cdot)-x_{0}(\cdot)\right) \rightarrow 0$ implies that the sequence $\left\{x_{n}(\cdot)-x_{0}(\cdot)\right\}$ contains a subsequence $\left\{x_{n_{k}}(\cdot)-x_{0}(\cdot)\right\}$ such that

$$
\sum_{k=1}^{\infty} \int_{\Omega} N\left(t,\left\|x_{n_{k}}(t)-x_{0}(t)\right\|_{X}\right) d \mu<+\infty
$$

Thus replacing the sequence $\left\{x_{n}(\cdot)-x_{0}(\cdot)\right\}$ by the subsequence $\left\{x_{n_{k}}(\cdot)-x_{0}(\cdot)\right\}$ we can assume without loss of generality that

$$
\sum_{n=1}^{\infty} \int_{\Omega} N\left(t,\left\|x_{n}(t)-x_{0}(t)\right\|_{x}\right) d \mu<+\infty
$$

Now we have the following two possibilities: either (1) $\mu(\Omega)$ is finite or (2) $\mu(\Omega)$ is infinite.

Case $(1): \mu(\Omega)$ is finite. We shall construct by induction a sequence of positive numbers $\left\{\varepsilon_{k}\right\}$, a sequence of measurable sets $\left\{\Omega_{k}\right\}\left(\Omega_{k} \subset \Omega\right)$ and a subsequence $\left\{x_{n_{k}}-\right.$ $\left.x_{0}\right\}$ such that the following conditions are satisfied:

(a) $\varepsilon_{k+1}<\frac{e_{k}}{2}$

(b) $\mu\left(\Omega_{k}\right) \leq \varepsilon_{k}$

(c) $\int_{\Omega_{k}} M\left(t, u_{n_{k}}(t)\right) d \mu>\frac{2}{3} r$

(d) $\int_{D} M\left(t, u_{n_{k}}(t)\right) d \mu<\frac{1}{3} r$ for any set $D \subset \Omega_{k}$ such that $\mu(D) \leq 2 \varepsilon_{k+1}$.

We put $\varepsilon_{1}=\mu(\Omega), x_{n_{1}}-x_{0}=x_{1}-x_{0}$ and $\Omega_{1}=\Omega$. Suppose that $\varepsilon_{k}, x_{n_{k}}-x_{0}$ and $\Omega_{k}$ have been constructed. Since $N_{F}$ is a Nemytskii set-valued operator mapping of the modular space $\left(N(L(\Omega, \Sigma, \mu ; X)), \rho_{N, \mu}\right)$ into subsets of the modular space $\left(M(L(\Omega, \Sigma, \mu ; Y)), \rho_{M, \mu}\right)$, by property $\left(\Delta_{2}\right)$,

$$
\int_{\mathbf{n}_{k}} M\left(t, u_{n_{k}}(t)\right) d \mu<+\infty
$$

Thus the function $M\left(t, u_{n_{k}}(t)\right)$ is absolutely continuous. Hence it is easy to find $\varepsilon_{k+1}$ satisfying conditions (a) and (d). Since the function $N=N(t, u)$ satisfies condition (A) and $\rho\left(x_{n}(\cdot)-x_{0}(\cdot)\right) \rightarrow 0$, the functions $x_{n}$ tend to $x_{0}$ in measure. Replacing eventually the sequence $\left\{x_{n_{k}}-x_{0}\right\}$ by its subsequence, we can assume without loss of generality that $x_{n_{k}}(t)$ tends to $x_{0}(t)$ almost everywhere. By the global lower semicontinuity of $F(t, x)$, we obtain that $d_{Y}\left(y_{n}(t), F\left(t, x_{n}(t)\right)\right)$ tends to 0 almost everywhere. 
Since $\mu(\Omega)<+\infty$, for the sequence $\left\{z_{n}(\cdot)\right\}$ of measurable selections chosen at the beginning of this proof such that (7), holds, there are an index $n_{k+1}$ and a set $E_{k+1} \subset \Omega$ such that, for $t \in E_{k+1}$,

$$
M\left(t, u_{n_{k}}(t)\right)<\frac{r}{3 \mu(\Omega)}
$$

and

$$
\mu\left(\Omega \backslash E_{k+1}\right)<\varepsilon_{k+1} .
$$

Let $\Omega_{k+1}=\Omega \backslash E_{k+1}$. Observe that (12) $)_{1}$ implies condition (b). By $(8)_{1}$ and $(11)_{1}$, we obtain

$$
\int_{\Omega_{k+1}} M\left(t, u_{n_{k+1}}(t)\right) d \mu=\int_{\Omega} M\left(t, u_{n_{k+1}}(t)\right) d \mu-\int_{E_{k+1}} M\left(t, u_{n_{k+1}}(t)\right) d \mu>\frac{2}{3} r .
$$

By properties (a) and.(b), we get

$$
\mu\left(\bigcup_{j=k+1}^{\infty} \Omega_{j}\right) \leq \sum_{j=k+1}^{\infty} \mu\left(\Omega_{j}\right) \leq \sum_{j=k+1}^{\infty} \varepsilon_{j}<2 \varepsilon_{k+1} .
$$

Thus we have constructed a sequence of positive numbers $\left\{\varepsilon_{k}\right\}$, a sequence of measurable sets $\left\{\Omega_{k}\right\}, \Omega_{k} \subset \Omega$, and a subsequence $\left\{x_{n_{k}}-x_{0}\right\}$ such that conditions (a) - (d) hold.

Now we shall continue the proof. Let

$$
D_{k}=\Omega_{k} \backslash\left(\bigcup_{j=k+1}^{\infty} \Omega_{j}\right) \quad(k \in I N)
$$

Define functions $\psi, \psi_{0}, y_{0}, z$ in the following way:

$$
\begin{aligned}
\psi(s) & = \begin{cases}x_{n_{k}}(s) & \text { if } s \in D_{k} \quad(k \in \mathbb{N}) \\
0 & \text { if } s \notin \cup_{j=1}^{\infty} D_{j}\end{cases} \\
\psi_{0}(s) & = \begin{cases}x_{0}(s) & \text { if } s \in D_{k} \quad(k \in \mathbb{N}) \\
0 & \text { if } s \notin \cup_{j=1}^{\infty} D_{j}\end{cases} \\
y_{0}(s) & = \begin{cases}y_{n_{k}}(s) & \text { if } s \in D_{k} \quad(k \in \mathbb{N}) \\
w(s) & \text { if } s \notin \cup_{j=1}^{\infty} D_{j}\end{cases} \\
z(s) & = \begin{cases}z_{n_{k}}(s) & \text { if } s \in D_{k}(k \in \mathbb{N}) \\
v(s) & \text { if } s \notin \cup_{j=1}^{\infty} D_{j}\end{cases}
\end{aligned}
$$

where $w(\cdot)$ and $v(\cdot)$ belong to $N_{F}(0)$ (i.e. these are measurable selections of $F(s, 0)$ ). From conditions (c), (d) and inequalities $(11)_{1},(13)_{1}$ it follows that

$$
\begin{aligned}
\int_{D_{k}} M\left(t,\left\|y_{0}(t)-z(t)\right\|\right) d \mu & =\int_{D_{k}} M\left(t, u_{n_{k}}(t)\right) d \mu \\
& =\int_{\Omega_{k}} M\left(t, u_{n_{k}}(t)\right) d \mu-\int_{\Omega_{k} \backslash D_{k}} M\left(t, u_{n_{k}}(t)\right) d \mu \\
& >\frac{1}{3} r .
\end{aligned}
$$


Observe that $\psi_{0} \in N(L(\Omega, \Sigma, \mu ; X))$ and, by $(10)_{1}$, also $\psi \in N(L(\Omega, \Sigma, \mu ; X))$. It is easy to see that $y_{0}(\cdot) \in N_{F}\left(\psi_{0}(\cdot)\right)$ and $z(\cdot) \in N_{F}(\psi(\cdot))$.

On the other hand,

$$
\int_{\Omega} M\left(t,\left\|y_{0}(t)-z(t)\right\|\right) d \mu \geq \sum_{k=1}^{\infty} \int_{D_{k}} M\left(t,\left\|y_{0}(t)-z(t)\right\|\right) d \mu=+\infty
$$

which contradicts the fact that $N_{F}$ is a set-valued operator mapping of the modular space $\left(N(L(\Omega, \Sigma, \mu ; X)), \rho_{N, \mu}\right)$ into subsets of the space $\left(M(L(\Omega, \Sigma, \mu ; Y)), \rho_{M, \mu}\right)$. This finishes the proof of the case when $\mu(\Omega)$ is finite.

Case (2): $\mu(\Omega)$ is infinite. We will consider the following two subcases:

(2a) There are a subset $\Omega_{0} \subset \Omega$ with finite measure $\mu\left(\Omega_{0}\right)$ and a number $\beta \in(0, r)$ such that $\int_{\Omega_{0}} M\left(t, u_{n}(t)\right) d \mu \geq \beta(n \in \mathbb{N})$.

(2b) There are a subsequence $\left\{u_{n_{k}}(t)\right\}$ and a sequence of measurable sets $\left\{D_{k}\right\}$ such that

(e) $\mu\left(D_{k}\right)<+\infty$ and $D_{i} \cap D_{j}=\emptyset$ for $i \neq j$

(f) $\int_{D_{k}} M\left(t, u_{n_{k}}(t)\right) d \mu \geq \frac{1}{2} r(k \in \mathbb{N})$.

In the subcase (2a) the consederation can be reduced to that of Case (1) with replacing $r$ by $\beta$. In the subcase (2b) we define functions $\psi, \psi_{0}, y, z$ by formulae $(14)_{1}-(17)_{1}$. As in Case (1) we obtain that $\psi, \psi_{0} \in N(L(\Omega, \Sigma, \mu ; X))$, and that $z(\cdot) \in N_{F}(\psi(\cdot))$ and simultaneously $y_{0}(\cdot) \in N_{F}\left(\psi_{0}(\cdot)\right)$. On the other hand, by properties (e) and (f) we obtain that $z(t)-y_{0}(t) \notin\left(M(L(\Omega, \Sigma, \mu ; Y)), \rho_{M, \mu}\right)$, which leads to a contradiction

Theorem 2. Let $X, Y$ be two separable $F$-spaces, $(\Omega, \Sigma, \mu)$ a measure space with complete non-atomic and $\sigma$-finite measue $\mu$, and $N_{F}$ a Nemytskiz set-valued operator mapping of a modular space $\left(N(L(\Omega, \Sigma, \mu ; X)), \rho_{N, \mu}\right)$ into subsets of a modular space $\left(M(L(\Omega, \Sigma, \mu ; Y)), \rho_{M, \mu}\right)$ induced by a sup-measurable set-valued function $F=F(t, u)$ : $\Omega \times X \rightarrow 2^{Y}$. Suppose that

(i) the function $N=N(t, u)$ satisfies condition (A)

(ii) the function $M=M(t, u)$ satisfies condition $\left(\Delta_{2}\right)$.

If the function $F=F(t, u)$ is lower semicontinuous with respect to $u$ for almost all $t \in \Omega$, then the operator $N_{F}$ is lower semicontinuous.

Proof. Suppose that the operator $N_{F}$ is not lower semicontinuous at some point $\left(x_{0}(\cdot), y_{0}(\cdot)\right)$ with $y_{0}(\cdot) \in N_{F}\left(x_{0}(\cdot)\right)$. This implies that there are a number $r>0$ and a sequence $\left\{x_{n}(\cdot)\right\}$ such that

$$
\begin{aligned}
& \rho_{N, \mu}\left(x_{n}(\cdot)-x_{0}(\cdot)\right) \rightarrow 0 \\
& \quad \inf _{z(\cdot) \in N_{F}\left(x_{n}(\cdot)\right)} \rho_{M, \mu}\left(z(\cdot)-y_{0}(\cdot)\right)>r \quad(n \in \mathbb{N}) .
\end{aligned}
$$


By [2: Theorem 8.24 and Corollary 8.23], for arbitrary $\eta>0$ there is a sequence $\left\{z_{n}(\cdot)\right\}$ of measurable selections $z_{n}(\cdot) \in N_{F}\left(x_{n}(\cdot)\right)$ such that, for almost all $t \in \Omega$,

$$
\left\|y_{0}(t)-z_{n}(t)\right\|_{Y}<(1+\eta) d_{Y}\left(y_{0}(t), F\left(t, x_{n}(t)\right)\right)
$$

where as before $d_{Y}(y, A)$ denotes the distance of a point $y$ to a set $A$ in the norm $\|\cdot\| Y$. We denote $u_{n}(t)=\left\|y_{0}(t)-z_{n}(t)\right\|_{Y}$. Since $z_{n}(\cdot) \in N_{F}\left(x_{n}(\cdot)\right)$, then

$$
\int_{\Omega} M\left(t, u_{n}(t)\right) d \mu \geq \inf _{z(\cdot) \in N_{F}\left(x_{n}(\cdot)\right)} \rho_{M, \mu}\left(z(\cdot)-y_{0}(\cdot)\right)>r \quad(n \in \mathbb{N}) .
$$

Then we continue the proof step by step in the same way as in the proof of Theorem 1 replacing $y_{n}(\cdot)$ by $y_{0}(\cdot)$. The only difference is to show the existence of a subsequence $\left\{u_{n_{k}}\right\}$ such that the inequalities

$$
M\left(t, u_{n_{k+1}}(t)\right)<\frac{r}{3 \mu(\Omega)}
$$

and

$$
\mu\left(\Omega \backslash E_{k+1}\right)<\varepsilon_{k+1}
$$

hold. In order to do this we replace the global lower semicontinuity of $\Gamma$ by its lower semicontinuity. Since the function $N=N(t, u)$ satisfies condition (A) and $\rho_{N, \mu}\left(x_{n}(\cdot)-x_{0}(\cdot)\right) \rightarrow 0$, the sequence $\left\{x_{n}(\cdot)\right\}$ tends to $x_{0}(\cdot)$ in measure. Thus by the lower semicontinuity of $F(t, x)$ with respect to $x, d_{Y}\left(y_{0}, F\left(t, x_{n}(t)\right)\right)$ tends to 0 in measure.

Since $\mu(\Omega)<+\infty$, for the sequence of measurable selections $\left\{z_{n}(\cdot)\right\}$ there are an index $n_{k+1}$ and a subset $E_{k+1} \subset \Omega$ such that, for $t \in E_{k+1}$, the inequalities

$$
M\left(t, u_{n_{k+1}}(t)\right)<\frac{r}{3 \mu(\Omega)}
$$

and

$$
\mu\left(\Omega \backslash E_{k+1}\right)<\varepsilon_{k+1}
$$

hold. The remained part of the proof can be continued in the same way as presented in the proof of Theorem 1

Theorem 3. Let $X, Y$ be two separable $F$-spaces, $(\Omega, \Sigma, \mu)$ a measure space with complete non-atomic and $\sigma$-finite measue $\mu$, and $N_{F}$ a Nemytskii set-valued operator mapping of a modular space $\left(N(L(\Omega, \Sigma, \mu ; X)), \rho_{N, \mu}\right)$ into subsets of a modular space $\left(M(L(\Omega, \Sigma, \mu ; Y)), \rho_{M, \mu}\right)$ induced by a sup-measurable set-valued function $F=F(t, u)$ : $\Omega \times X \rightarrow 2^{Y}$. Suppose that

(i) the function $N=N(t, u)$ satisfies condition (A)

(ii) the function $M=M(t, u)$ satisfies condition $\left(\Delta_{2}\right)$.

If the function $F=F(t, u)$ is metric upper semicontinuous with respect to $u$ for almost all $t \in \Omega$, then the operator $N_{F}$ is metric upper semicontinuous. 
Proof. Suppose that the operator $N_{F}$ is not metric upper semicontinuous. This implies that there are a number $r>0$ and sequences $\left\{x_{n}(\cdot)\right\}$ and $\left\{y_{n}(\cdot)\right\}$ with $y_{n}(\cdot) \in$ $N_{F}\left(x_{n}(\cdot)\right)$ such that

$$
\begin{aligned}
& \rho_{N, \mu}\left(x_{n}(\cdot)-x_{0}(\cdot)\right) \rightarrow 0 \\
& \quad \inf _{z(\cdot) \in N_{F}\left(x_{0}(\cdot)\right)} \rho_{M, \mu}\left(z(\cdot)-y_{n}(\cdot)\right)>r \quad(n \in \mathbb{N}) .
\end{aligned}
$$

By [2: Theorem 8.24 and Corollary 8.23], for arbitrary $\eta>0$ there is a sequence $\left\{z_{n}(\cdot)\right.$ \} of measurable selections $z_{n}(\cdot) \in N_{F}\left(x_{0}(\cdot)\right)$ such that, for almost all $t \in \Omega$,

$$
\left\|y_{n}(t)-z_{n}(t)\right\|_{Y}<(1+\eta) d_{Y}\left(y_{n}(t), F\left(t, x_{0}(t)\right)\right)
$$

where as before $d_{Y}(y, A)$ denotes the distance of a point $y$ to a set $A$ in the norm $\|\cdot\| Y$. We denote $u_{n}(t)=\left\|y_{n}(t)-z_{n}(t)\right\|_{Y}$. Since $z_{n}(\cdot) \in N_{F}\left(x_{0}(\cdot)\right)$, then

$$
\int_{\Omega} M\left(t, u_{n}(t)\right) d \mu \geq \inf _{z(\cdot) \in N_{F}\left(x_{0}(\cdot)\right)} \rho_{M, \mu}\left(z(\cdot)-y_{n}(\cdot)\right)>r \quad(n \in \mathbb{N}) .
$$

Then we continue the proof step by step in the same way as in the proof of Theorem 1. The only difference is to show the existence of a subsequence $\left\{u_{n_{k}}\right\}$ such that the inequalities

$$
M\left(t, u_{n_{k+1}}(t)\right)<\frac{r}{3 \mu(\Omega)}
$$

and

$$
\mu\left(\Omega \backslash E_{k+1}\right)<\varepsilon_{k+1}
$$

hold. In order to do this we replace the global lower semicontinuity of $\Gamma$ by its metric upper semicontinuity. Since the function $N=N(t, u)$ satisfies condition (A) and $\rho_{N, \mu}\left(x_{n}(\cdot)-x_{0}(\cdot)\right) \rightarrow 0$, the sequence $\left\{x_{n}(\cdot)\right\}$ tends to $x_{0}(\cdot)$ in measure. Thus by the metric upper semicontinuity of $F(t, x)$ with respect to $x, d_{Y}\left(y_{n}, F\left(t, x_{0}(t)\right)\right)$ tends to 0 in measure.

Since $\mu(\Omega)<+\infty$, for the sequence of measurable selections $\left\{z_{n}(\cdot)\right\}$ there are an index $n_{k+1}$ and a subset $E_{k+1} \subset \Omega$ such that, for $t \in E_{k+1}$, the inequalities

$$
M\left(t, u_{n_{k+1}}(t)\right)<\frac{r}{3 \mu(\Omega)}
$$

and

$$
\mu\left(\Omega \backslash E_{k+1}\right)<\varepsilon_{k+1}
$$

hold. The remained part of the proof can be continued in the same way as presented in the proof of Theorem 1

Theorem 3 generalizes Theorem 1 of [3], where it is proved for Banach spaces $X, Y$ and for functions $N(t, u)=u^{p}, M(t, u)=u^{q}$ with $1 \leq p \leq q<+\infty$ under some estimation assumptions warranting that a Nemytskii operator $N_{F}$ induced by a supmeasurable set-valued function $F=F(t, u)$ maps the space $L^{p}(\Omega, \Sigma, \mu ; X)$ into the space $L^{q}(\Omega, \Sigma, \mu ; Y)$. 


\section{References}

[1] Appell, J., Nguyen, H. T. and P. P. Zabrejko: Multivalued superposition operators in ideal spaces of vector functions. Parts I and II. Indag. Math. (N. S.) 2 (1991), $385-395$ and $397-409$.

[2] Aubin, J. P. and H. Frankowska: Set-Valued Analysis (Systems and Control: Vol. 2). Boston et al: Birkbäuser Verlag 1990.

[3] Cellina, A., Fryszkowska, A. and T. Rzezuchowski: Uppersemicontinuity of Nemytskij operalors. Ann. Mat. Pura Appl. 160 (1991), 321 - 330.

[4] Goncharow, V. V.: On the existence of solutions of a class of differential inclusions on a compact set (in Russian). Sib. Math. Zhur. 31 (1990)5, 24 - 30.

[5] Musielak, J.: Orlicz Spaces and Modular Spaces. Lect. Notes Math. 1034 (1983), 1 - 222.

[6] Musielak, J. and W. Orlicz: On modular spaces. Stud. Math. 18 (1959), 49 - 65.

[7] Nakano, H.: Modulared linear spaces. J. Fac. Sci. Univ. Tokyo (Section I) 6 (1950), 85 131.

[8] Nakano, H.: Modulared Semi-Ordered Linear Spaces. Tokyo: Maruzen Co. 1950.

[9] Nakano, H.: Topology and Linear Topological Spaces. Tokyo: Maruzen Co. 1951.

[10] Niemytzki, V.: Sur les équations integrales non-linéaires. C. R. Acad. Sci. Paris 196 (1933), $836-838$.

[11] Niemytzkij, V.: Théorèmes d'existence et d'unicite des solutions de quelques équations integrales non-linéaires. Matem. Sbornik 41 (1934), 421 - 438.

[12] Rolewicz, S.: Metric Linear Spaces. Dortrecht: D. Reidel 1985, and Warszawa: Polish Sci. Publ. 1985. 